\title{
Diagnostic and Prognostic Values of Interferon Inducible Protein 10 in Occurrence and Outcome of Sepsis in Preterm Neonates
}

\author{
Elsayed Ahmed Goda, Mohammed Shees Ahmed Shahhat, Mohammed Mahmood Abd \\ Elmohsen, Mohammed Saad Ahmed, Bahaa Mohammed Ahmed, \\ Mohammed Mustafa Mohammed Abdu
}

\author{
Department of Microbiology, Faculty of Medicine, Al Azhar University \\ Corresponding author: Mohammed Mustafa Mohamed Abdo, Mobile: 00201009319260, E-Mail: \\ medo22121987@gmail.com
}

\begin{abstract}
:
Background: Infection is still an important cause of neonatal morbidity and mortality despite development of broad-spectrum antibiotics and clinical advances in life support therapy.

Aim of the work: To evaluate plasma interferon gamma inducible protein 10 (IP-10) in the diagnosis and prognosis of sepsis in preterm neonates and comparison with CRP and blood culture.

Subjects and methods: Samples collected from 40 preterm neonates (20 with suspected infection and 20 with no suspicion of infection) from NICU of Elsayed Galal Hospital were subjected to blood culture, $\mathrm{CRP}, \mathrm{CBC}$ and measurement of plasma interferon induced protein 10 using sandwich enzyme linked immunosorbent assay (ELISA). Results: The study recruited 40 neonates divided into two groups (20 neonates each). In our study, the mean gestational age was 32.7 weeks for patient cases. The mean birth weight was $1.557 \mathrm{~kg}$ for confirmed cases. CRP was found to be higher in septic than non-septic group with a range from 6 to $24 \mathrm{mg} / \mathrm{dl}$. The blood culture in our study was positive in 18 cases (11 cases had klebsiella, 2 had acinetobacter, 2 had coagulase negative staph (cons), one had staph aureus, one had pseudomonas, and one had MRSA) while others were negative. In our study, IP-10 cut-off for diagnosing sepsis was $133 \mathrm{ng} / \mathrm{ml}$. Our data revealed that there was significantly higher plasma IP-10 levels in preterm neonates with neonatal sepsis compared to healthy neonates. Conclusion and Recommendations: The level of IP-10 increased early in cases with suspicion of infection. Plasma IP10 is a valuable laboratory test in the assessment of preterm infants aged $<1$ week with suspicion of sepsis and may serve as a better diagnostic marker of sepsis than CRP.
\end{abstract}

Keywords: IP10, neonatal sepsis, Preterm and full term.

\section{Introduction}

Early-onset sepsis remains a common and serious problem for neonates, especially preterm infants. The diagnosis of neonatal sepsis is based on a combination of clinical presentation; the use of nonspecific markers, including C-reactive protein and pro calcitonin (where available), blood cultures, and the use of molecular methods, including PCR. Cytokines, including interleukin 6 (IL-6), interleukin 8 (IL8), gamma interferon (IFN- $\gamma$ ), tumor necrosis factor alpha (TNF- $\alpha$ ) and cell surface antigens, including soluble intercellular adhesion molecule (sICAM) and CD64, are also being increasingly examined for use as nonspecific screening measures for neonatal sepsis (1). Immune assay of serum cytokines and chemokines can potentially be used as a marker of infection for both diagnostic and prognostic purposes $^{(2)}$.

Interferon-inducible protein-10 is also called gamma-IP-10 or INP-10. It has a length of 98 amino acids and belongs to the family of chemotactic cytokines. IP-10 has been detected in keratinocytes, lymphocytes, monocytes, and endothelial cells in immunologically mediated processes ${ }^{(3)}$.

Preterm infants who developed necrotizing enterocolitis (NEC) had threefold increased risk of mortality. As the immunologic defense of preterm infants is considered to be immature and or deficient, this category of patients is particularly vulnerable to developing severe and opportunistic infections in the immediate postnatal period. However, early warning signs and symptoms of systemic infection and NEC are often nonspecific, inconspicuous and can easily be confused with non-infective causes such as apnea of prematurity, gastrointestinal dysmotility, and acute exacerbation of bronchopulmonary dysplasia $^{(4,5,6)}$. 
Laboratory indicators, such as complete blood-cell count (CBC), ratio of immature to total neutrophil and C-reactive protein (CRP) do not have high sensitivity especially if measured early in the course of sepsis ${ }^{(7)}$. Thus, early diagnosis of infants with bacterial sepsis and NEC has been recognized to be a major diagnostic challenge ${ }^{(8)}$.

Exposure to microorganisms and their derived products triggers a rapid and coordinated sequence of host reactions resulting in recruitment of leucocytes into areas of inflammation or sites of microbial invasion (9).

\section{Aim of the Work}

To evaluate plasma interferon gamma inducible protein 10 (IP-10) in the diagnosis and prognosis of sepsis in preterm neonates and comparison with CRP and blood culture.

\section{Subjects and Methods}

A- Subjects: This cross-sectional-case control study was carried out in Neonatal Intensive Care Unit, Elsayed Galal University Hospital from May to November 2017. It was conducted on forty (40) neonates. The study was approved by the Ethics Board of AlAzhar University.

\section{They were divided into two groups:}

1- Group (1): included (20) preterm neonates with suspected early infected sepsis (patient).

2- Group (2): included (20) preterm neonates with no suspicion of sepsis(control)

Neonates of both groups were subjected to the following:

- History taking: prenatal, natal and family history laying stress on sex, birth weight, gestational age, postnatal age and mode of delivery.

- Complete clinical examination laying stress on gestational age, temperature, tolerance to oral feeding, abdominal distension, residual gastric aspirate, cyanosis and others .

\section{B- Methods:}

- Laboratory investigation: in the form of:

On admission:

Interferon gamma inducible protein10 level was measured using sandwich enzyme linked immunosorbent assay (ELISA) .(First sample)

Then all cases underwent the following investigation:

1- Complete blood picture with differential count.

2-CRP (Quantitative), equals to or more than $8 \mathrm{mg} / \mathrm{dl}$ is considered a case.

\section{3-Blood culture}

Procedure:

1-Blood sample $(1 \mathrm{ml})$ was collected by open cannula 'syringe' technique; using a sterile cannula and syringe and aspirate the blood repeatedly. The cannula was then being left in place for antibiotics.

2-Infant's blood samples were transferred immediately into the blood culture bottles by puncturing the rubber stopper with the needle, then the needle was discarded and the contents were mixed gently by inverting the bottle $2-3$ times.

3- Blood culture bottles were inverted for allowing broth to flood the agar surface and incubated at $35^{\circ} \mathrm{C}$ for 7 days. The bottles were inverted every day and observed for any growth on the agar surface.

\section{- Isolates identification:}

Isolates obtained were identified by standard microbiological techniques, namely, motility, Gram staining, colony characteristics, and biochemical properties using API system.

Antimicrobial-susceptibility testing (Disk diffusion method):

- 3-5 well isolated colonies of similar appearance to the organism were removed using a sterile wire loop and emulsified in sterile physiological saline.

- A good light Inoculum's suspension was prepared matched to the turbidity standard ( 0.5 McFarland).

- The plate of Muller-Hinton agar was inoculated with the prepared suspension by a sterile swab and allowed to dry for 3to5min.

- The antibiotic-discs were placed on the surface of the inoculated plates using a sterile forceps, and were gently pressed down onto the agar to ensure complete contact to the surface.

- Arrangement of the discs was done as they were at $15 \mathrm{~mm}$ from edge of the plate and $25 \mathrm{~mm}$ from disc to disc.

- The plates were inverted and incubated aerobically at $35^{\circ} \mathrm{C}$. 
- After (18-24hrs) of incubation, the plates were examined, and the diameter of the zones of complete inhibition of the growth was measured, by use of a ruler.

- The end point used was complete inhibition of growth around the disk.

- The zone diameter for individual antibiotic was translated into susceptible, moderately susceptible, or resistant categories by interpretation chart.

4- Interferon gamma inducible protein- 10 level was measured using sandwich enzyme linked immunosorbent assay ELISA).

(a)-Specimen collection:

$1 \mathrm{ml}$ blood collected from each subject and added to tube containing EDTA. Plasma was separated by centrifugation (1900 rpm for $5 \mathrm{~min})$ at $4^{\circ} \mathrm{C}$ and stored at $-80^{\circ} \mathrm{C}$.

\section{assay Procedure:}

1-.Dilution of the standard: 50 ul standard dilution was added to each tube.

100ul standard $(135 \mathrm{ng} / \mathrm{L})$ added to the first tube. In addition, $100 \mathrm{ul}$ taken from the first tube into the second tube. 50 ul from the second tube taken to the third tube and dilution series produced.

2- Preparing sample: in blank wells separated 40 ul sample dilution were added to test sample wells. Then, $10 \mathrm{ul}$ sample was added to each well, and mix gently.

3- Incubation: Cover with the adhesive strip and incubation for $30 \mathrm{~min}$ at room temperature was done.

4- Dilution of dilute solution with distilled water was done: $100 \mathrm{~mL}$ of Wash Buffer Concentrate (provided) was diluted into RESULTS deionized or distilled water to prepare $1000 \mathrm{~mL}$ of Wash Buffer.

5- Washing:

- Liquid was removed from the wells by aspirating the plate

- Excess liquid was removed by grasping the plate firmly and smartly rapping the inverted plate on a clean paper towel at least 5 times.

- Each well was filled with $400 \mathrm{uL}$ of Wash Buffer using a squirt bottle.

- Liquid was removed from the wells by inverting the plate and decanting the Contents.

6- Adding enzyme: $200 \mathrm{uL}$ of IP-10 Conjugate which is monoclonal antibody against IP-10 conjugated to horseradish peroxidase was added to each well. Cover with a new adhesive strip was done. Incubation was done for 2 hours at room temperature.

7- Washing: as step 5.

8- 50 ul of chromogen solution $\mathrm{A}$ and chromogen solution B were added to each well, avoid light, preservation for $15 \mathrm{~min}$ at room temperature

9- $50 \mathrm{ul}$ of stop solution were added to each well, stop the reaction (the blue changed to yellow).

10- Taking blank well as zero, the optical density was determined for each well within 30 minutes, using a micro plate reader set to 450 $\mathrm{nm}$.

5-Interferon gamma inducible protein- 10 level was measured again using sandwich enzyme linked immunosorbent assay ELISA) 7days after treatment

-Statistical analysis was done according to (Warne RT et al., 2012).

Table (1): Comparison of demographic and fetal risk factors between studied groups

\begin{tabular}{|l|l|l|l|l|}
\hline Demographic and fetal risk factors & Patient & Control & P value & Sig. \\
\hline $\begin{array}{l}\text { Sex (no. \%) } \\
\text { Male } \\
\text { Female }\end{array}$ & $\begin{array}{l}13(66.7) \\
7(33.3)\end{array}$ & $\begin{array}{l}13(66.7) \\
7(33.3)\end{array}$ & 0.635 & NS \\
\hline $\begin{array}{l}\text { Gestational age (Weak) } \\
\text { Mean } \pm \text { SD }\end{array}$ & $32.7 \pm 3.6$ & $33.4 \pm 3.5$ & 0.525 & NS \\
\hline $\begin{array}{l}\text { Weight (gm.) } \\
\text { Mean } \pm \text { SD }\end{array}$ & $1557.3 \pm 823.2$ & $1840.0 \pm 631.1$ & 0.007 & S \\
\hline $\begin{array}{l}\text { Stay in NICU (Day) } \\
\text { Mean } \pm \text { SD }\end{array}$ & $9.8 \pm 6.6$ & $3.3 \pm 3.1$ & 0.001 & HS \\
\hline
\end{tabular}

SD: Standard deviation

S: P-value $<0.05$ (Significant) HS: P-value $<0.01$ (High significant)

P-value > 0.05 (Non-significant)

This table showed comparison of demographic data and fetal risk factors between patient and control groups which was not significant except for Stay in NICU (more in patient than control) and weight (more in control than patient). 
Diagnostic and Prognostic Values of Interferon Inducible Protein 10 in Occurrence...

Table (2): Comparison between patient and control as regarding laboratory findings

\begin{tabular}{|l|l|l|l|l|}
\hline & $\begin{array}{l}\text { Normal }(\mathrm{n}=2) \\
\text { Mean } \pm \text { SD }\end{array}$ & $\begin{array}{l}\text { Abnormal }(\mathrm{n}=25) \\
\text { Mean } \pm \text { SD }\end{array}$ & P value & Sig. \\
\hline $\mathrm{Hb}(\mathrm{gm} / \mathrm{dl})$ & $12.2 \pm 0.7$ & $14.8 \pm 2.2$ & $\mathbf{0 . 1 1 1}$ & NS \\
\hline WBC & $20.0 \pm 2.0$ & $11.7 \pm 9.8$ & 0.248 & NS \\
\hline Platelet & $184.0 \pm 17.0$ & $137.9 \pm 105.2$ & 0.547 & NS \\
\hline
\end{tabular}

This table showed no significant difference between patient and control groups as regarding laboratory findings.

Table (3): Delivery risk factors of patient

\begin{tabular}{|l|l|l|}
\hline & No. & $\%$ \\
\hline PROM & 6 & 20 \\
\hline Hypertension & 3 & 10 \\
\hline
\end{tabular}

This table showed the most delivery risk factors of patient where PROM was $20 \%$ and hypertension was $10 \%$.

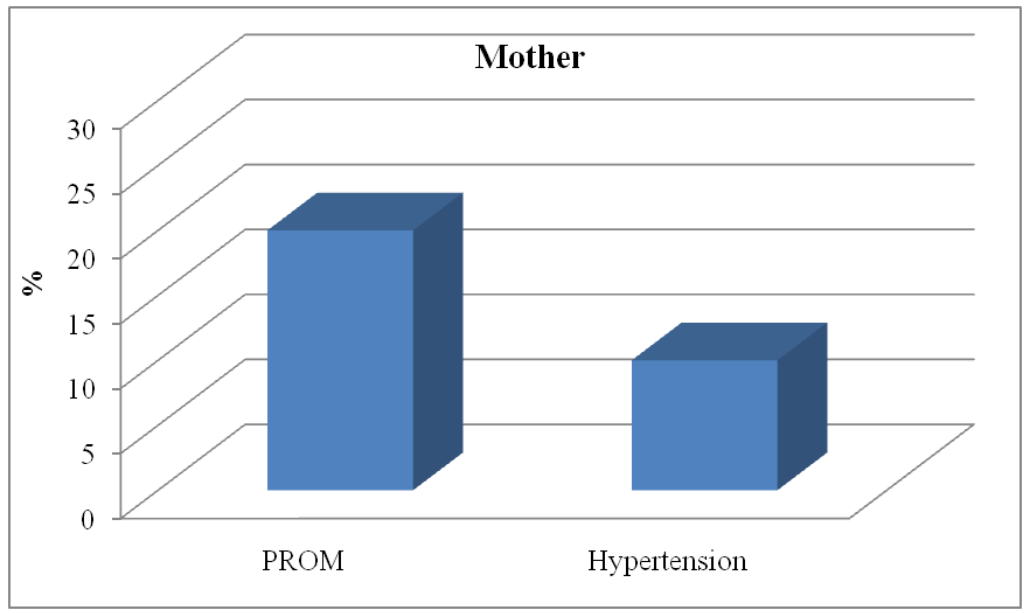

Figure (1): Delivery risk factors in patrients.

Table (4): Clinical picture of patient

\begin{tabular}{|l|l|l|}
\hline & No. & $\%$ \\
\hline Tachycardia & 22 & 56.7 \\
\hline Bradycardia & 4 & 10 \\
\hline Poor skin color & 25 & 73.3 \\
\hline Poor perfusion & 25 & 73.3 \\
\hline Apnea (Resp. system examination) & 9 & 23.3 \\
\hline Tachypnea & 20 & 50 \\
\hline Cyanosis & 13 & 33.3 \\
\hline Hypotonia & 11 & 26.7 \\
\hline Seizures & 8 & 20 \\
\hline Irritability & 12 & 30 \\
\hline Poor feeding & 40 & 100 \\
\hline Abdominal distention & 24 & 63.3 \\
\hline Hypothermia & 23 & 60 \\
\hline
\end{tabular}


This table showed that poor feeding was more frequent among patients (100\%) and RD (93.3\%), while Bradycardia was low frequent among patients (10\%).

Table (5): Blood culture in the studied groups.

\begin{tabular}{|l|l|l|l|}
\hline & & patient & Control \\
\hline \multirow{5}{*}{ Blood culture } & Nogrowth & 2 & 17 \\
\cline { 2 - 4 } & Klebsiella & 11 & - \\
\cline { 2 - 4 } & Acinetobacter & 2 & - \\
\cline { 2 - 4 } & Staph aureus & 1 & 1 \\
\cline { 2 - 4 } & Pseudomonus & 1 & 2 \\
\cline { 2 - 4 } & MRSA & 1 & - \\
\cline { 2 - 4 } & Cons & 2 & - \\
\hline
\end{tabular}

This table showed that Klebsiella was the most frequent among patients (53.3\%) followed by Acinetobacter $(16.7 \%)$.

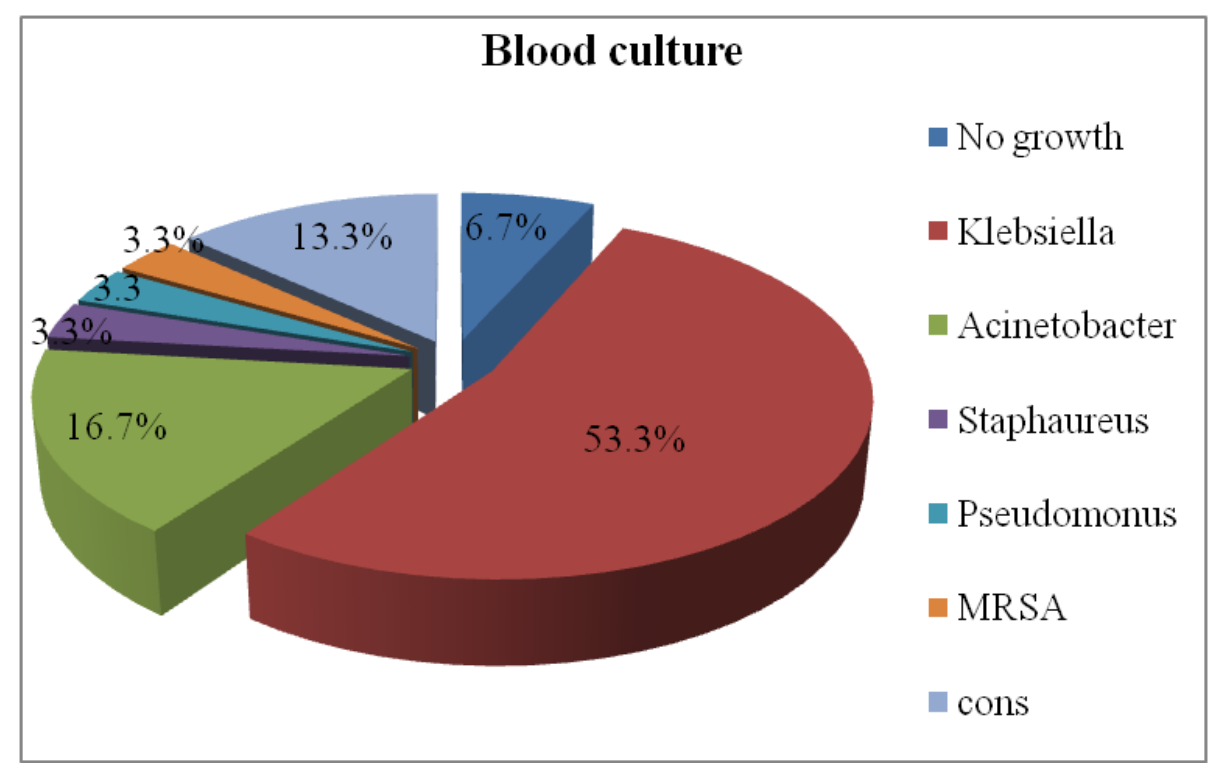

Figure (2): Blood culture in the patient.

Table (6): Level of IP 10 and CRP in the studied groups.(on admission)

\begin{tabular}{|l|l|l|l|l|}
\hline & Patient & Control & P value & Sig. \\
\hline IP-10 $(\mathrm{ng} / \mathrm{ml})$ & $333(0-1200)$ & $40(0-230)$ & 0.0001 & S \\
\hline CRP $(\mathrm{ug} / \mathrm{ml})$ & $24(0-96)$ & $6(0-12)$ & 0.62 & NS \\
\hline
\end{tabular}

SD: Standard deviation

P-value $<0.05$ (Significant)

P-value $>0.05$ (Non-significant)

This table showed that there was a significant difference between patient and control regarding IP-10 (high in patient than control) and there was no significant difference between patient and control regarding CRP.

Table (7): Level of IP10 and CRP in the studied groups.(7days after treatment).

\begin{tabular}{|l|l|l|l|c|}
\hline & Patient & Control & P value & Sig. \\
\hline IP-10(ng/ml) & $25(0-200)$ & $10(0-50)$ & 0.0001 & S \\
\hline CRP(ug/ml) & $12(0-24)$ & $6(0-12)$ & 0.62 & NS \\
\hline
\end{tabular}

SD: Standard deviation

P-value $<0.05$ (Significant)

P-value $>0.05$ (Non-significant)

This table showed that levels of IP-10 and CRP decreased in patient and control. 


\section{Discussion}

Despite improved neonatal care over the past decades, infections remain common and sometimes life-threatening in neonates admitted to the neonatal intensive care unit (NICU) and young infants ${ }^{(11)}$.

Chemokines are a large group of proteins that are classified on the basis of their chemical structure as $\mathrm{CXC}, \mathrm{CC}, \mathrm{C}$ and $\mathrm{CX} 3 \mathrm{C}$ chemokines. The chemokines are proinflammatory proteins that can accentuate the functions of many different WBCs. chemokines play a major role in disease in which there is an accentuated inflammatory component and they could assist migration and chemotaxis. It is reasonable that chemokine's increase in early phase of infection. Among CXC chemokines, IP10 was initially identified as early response gene induced by interferon gamma ${ }^{(12)}$. IP10 is secreted by several cell types including endothelial cells, keratinocytes, fibroblasts, monocytes and neutrophils (12). Chemokine IP10 has been identified to play an important role during infectious and inflammatory process such as chemo attraction for monocytes and $\mathrm{T}$ cells or promotion of $\mathrm{T}$ cell adhesion to endothelial cells and in TH1-type inflammatory diseases ${ }^{(13)}$. The study recruited 40 neonates divided in to two groups (20 patient and 20 control). In our study the mean gestational age was 32.7 weeks for patient cases. The mean birth weight was $1.557 \mathrm{~kg}$ for confirmed cases. By statistical analysis there were no significant differences between the groups regarding gestational age and birth weight. The sex of studied groups was in the confirmed sepsis 13 males and 7 females, and in the non-septic group 13 males and 7 females. By statistical analysis there was no statistical difference between the two groups regarding the sex (p value 0.635 ). There was a difference regarding weight ( $\mathrm{p}$ value 0.007 ) of statistical significance and this comes in agreement with Pak et al (14) who stated that there was no significant difference in clinical characteristics between infected and non-infected group as regard sex. Also in the study of Sallam et al. ${ }^{(15)}$ and Chen et al. (16), where they demonstrated that neonates with sepsis were older than healthy ones, with p-value $=0.01$, But, there was no significant difference in sex and weight between both groups. On the other hand, it was observed in other studies by De Benedetti et al. ${ }^{(17)}$ and Gomella et al. ${ }^{(18)}$ that lower birth weight is significantly associated with increased frequency of sepsis as we observed in our study that the relation between weight and sepsis is highly significant ( $\mathrm{p}$ value 0.007). This observation was due to the fact that the most important neonatal factor predisposing to infection is prematurity or low birth weight as the preterm infant has a 3-10 fold higher incidence of infection than full term normal birth weight infant ${ }^{(19)}$. Also, our study disagreed with the study of Gerdes (20) who found that the frequency of neonatal sepsis was significantly higher in males. Also, Rehan ${ }^{(21)}$ reported that male infants have a fourfold increased risk to develop sepsis than females as we found that frequency of sepsis in males double the frequency in females. In our study IP-10 was found to be higher among septic group than non-septic group. This comes in agreement with Sallam et al. ${ }^{(15)}$ who confirmed that plasma chemokine concentrations were significantly higher in serious bacterial infection (SBI) group than non-SBI group. Also, our study comes in agreement with Pak et al. (14) who found that IP-10 and other chemokine's (IL8-MIG and MCP-1) were significantly higher in infected group than noninfected group in the initial evaluation of sepsis in preterm neonates. In our study, CRP was found to be higher in septic than in non-septic group with a range from 6 to $96 \mathrm{mg} / \mathrm{dl}$. This comes in agreement with the study of Carrigan et al. ${ }^{(22)}$ who reported that concentrations of CRP in septic neonates range from 12 to 159 $\mathrm{mg} / \mathrm{ml}$, this small difference in the upper and lower limits of CRP was due to differences in laboratory techniques. On the other hand, Pak et al. ${ }^{(14)}$ and Sallam et al. ${ }^{(15)}$ confirmed that CRP is not a very sensitive early marker of infection. This can be attributed to that CRP is synthesized within 6 to 8 hours of exposure to an infective process or tissue damage. It has a half-life of 19 hours and may increase more than 1000 fold during an acute phase response. The blood culture in our study was positive in 18 cases (11 cases had klebsiella, 2 had acinetobacter, 2 had coagulase negative staph (cons), one had staph aureus, one had pseudomonas and one had MRSA) while others were negative. This can be attributed to the early use of antibiotics in early clinical suspicion. This comes in agreement with Hilgendorff et al. ${ }^{(23)}$ who said that it is now 
accepted widely that early onset neonatal sepsis is often a clinical diagnosis because blood cultures can be false negative after antenatal administration of antibiotics. Repeated blood cultures are needed for giving accurate diagnosis. Another cause may be due to that the blood sample taken from the baby may not be sufficient for the culture or wrong maneuver ${ }^{(24,}$ ${ }^{25)}$. In the current study, blood cultures were positive in about $90 \%$ of cases, but non similar results have been found in the study of Procianoy and silveire ${ }^{(26)}$. They found that blood cultures were positive in only 18 of total 85 cases $(21 \%)$ of neonatal sepsis. High blood concentration of IP-10 was high positively correlated with the severity of the infection process and the occurrence of septic shock and multisystem organ failure and death and then signified a poor progress ${ }^{(27)}$. In our study, IP10 cut-off for diagnosing sepsis was $133 \mathrm{ng} / \mathrm{ml}$. This comes in agreement with sallam et al. ${ }^{(15)}$ who studied the role of IP-10 as a predictor of serious bacterial infection in neonates and young infant. Pak et al. (14) studied the relationship of IP-10 and late onset bacterial sepsis and NEC in preterm infants. The cut-off for plasma IP-10 was $1250 \mathrm{pg} / \mathrm{ml}$ with sensitivity of $80 \%$ and specificity of $75 \%$ at 0 hour. This difference in IP-10 level in our study and this study is due to the use of different type of kits (cytometric bead array kits) and the method used for analysis (flow cytometry) while we used human IP-10 ELISA kits (Immuuneassay Quantikine kits). In other studies done by Chen et al. ${ }^{(16)}$ where they studied the role of plasma IP-10 as a predictor of serious bacterial infection in neonates and young infants, the IP-10 cut-off value was 48.2 $\mathrm{pg} / \mathrm{ml}$. Our data revealed that there were significantly higher plasma IP-10 levels in preterm neonates with neonatal sepsis compared to healthy neonates.

\section{Conclusion}

The level of IP-10 increased early in cases with suspicion of infection. Plasma IP-10 is a valuable laboratory test in the assessment of preterm infants aged $<1$ week with suspicion of sepsis and may serve as a better diagnostic marker of sepsis than CRP.

\section{Recommendations}

We recommend the following:

1- Adding IP-10 to the panel of diagnostic tools of neonatal sepsis, as it showed superiority in diagnosing preterm and term neonatal sepsis compared to other laboratory parameters that are commonly used (CBC and CRP). It is a simple, rapid and early detecting tool.

2- Future Studying of the level of IP-10 in different disease processes of neonates as jaundice, respiratory distress syndrome and transient tachypnea of newborn is needed.

\section{References}

1. Simonsen kA, Anderson-berry AL, Delair SF, Davies HD (2014): Early onset neonatal sepsis. American Society for Microbiology,27 (1): 2147.

2. Pak C, Lam HS (2006): Diagnostic markers for neonatal sepsis. Curr. Opin., 18 (2): 125-31.

3. Gottlieb AB, Luster AD, Posnett DN , Carter DM (1988): Detection of a gamma interferon-induced protein, IP10 , in psoriatic plaques. Journal of Experimental Medicine, 168 (3): 941948.

4. Siu yk, NG PC, Fung SC et al. (1998): Double blind, randomized, placebo controlled study of oral vancomycin in prevention of necrotizing enterocolitis in preterm, very low birth-weight infants. Arch. Dis. Child Fetal Neonatal, 79 (2): F105-F109.

5. Stoll BJ, Hansen N, Fanaroff AA et al. (2002): Late-onset sepsis in very low birth weight neonates: the experience of the NICHD Neonatal ResearchNetwork. Pediatrics, 110 (1): 285-291

6. Pak C (2005): Clinical trials for evaluating diagnostic markers of infection in neonates. Biol. Neonate, 87 (2): 111-2.

7. Kuster H, weiss M, WIlleitner AE et al. (1998): interleukin 1 receptor antagonist and interlukin-6 for early diagnosis of neonatal sepsis 2 days before clinical manifestation. Lancet, 352 (9136): 1271-1277.

8. Pak C, Li K, Leung TF et al. (2006): Early prediction of sepsis-induced disseminated intravascular coagulation with interleukin-10, interleukin-6, and RANTES in preterm infants. Clin. Chem., 52 (6): 1181-9.

9. Cavaillon JM, Adib-Conquy M, Fitting C et al. (2003): Cytokine 
cascade in sepsis. Scand J Infect Dis., 35 (9): 535-44.

10. Warne RT, Lazo M, Ramos T, Ritter N (2012): statistical methods used in Gifted Education Journals, Gifted child Quarterly, 56 (3): 134-149.

11. Stoll B, Weisman L (2005): Infections in perinatology. Clin Perinatol., 111 (24): 190-98.

12. Luster AD, Unkeless JC, Ravetech JV (1985): Gamma Interferon transcriptionally regulates an early response gene containing homology to platelet protiens. Nature, 315 (6021): 672-6.

13. Manes TD, Pober JS, Kluger MS (2006): Endothelial cell-T lymphocytes interactions: Ip-10 stimulates rapid trans-endothelial migration of human effector but not central memory CD4+ $\mathrm{T}$ cells. requirements for shear stress and adhesion molecules. Transplantation, 82 (1): 9-14.

14. Pak C, Li K, Chui KM et al. (2007): IP 10 is an early diagnostic marker for identification of late onset bacterial infection in preterm infants. Pediatr. Res., 61 (1): 93-8.

15. Sallam SA, Babrs GM, Said M, Taghian HM (2012): Plasma IP-10 as apredictor of serious bacterial infection in neonates and young infants. $\mathbf{J}$ American science, 8 (4): 190-194.

16. Chen HL, Hung CH ,TSENG HI, Yang RC (2011): plasma IP-10 as apredictor of serious bacterial infection in infants less than 4 months of age. Journal of tropical pediatrics, 57 (2): 145-151.

17. De bendetti F, Auriti C, Durbano LE et al. (2007): low serum levels of mannose binding lectin are risk factor for neonatal sepsis. Pediatr Res., 61 (3): 325-328

18. Gomella T, Cunningham M, Eyal F, Zenk K (2013): Neonatal Sepsis. In:
Clinical manual of neonatology. Neonatology management procedures on call problems, diseases and drugs, Gomella T.L (6th ed). Appleton Lange.

19. Karen JM, Robert MK (2018): Nelson essential of pediatrics. ,8th edition,https://inspectioncopy.elsevier. com/3/en/book/details/978032351145 2

20. Gerdes JS (2004): Diagnosis and management of bacterial infection in the neonates. Ped Clin North Am., 51 (4): 939-959.

21. Rehan VK, Moddemann D, Casiro OG (2002): Outcome of very low birth weight ( $<1500$ grams) infants born to mothers with diabetes. Clin. Pediatr., 41 (7): 481-91.

22. Carrigan SD, Scott G, Tobrizian $M$ (2004): Toward resolving the challenges of sepsis diagnosis. Clinical Chemistry, 50: 1301-1314.

23. Hilgendorff A, Schmidt R, Bohnert A et al. (2005): Host defence lectins in preterm neonates. Acta pediatr., 94 (6): $794-9$.

24. Joseph A, Prats J, Cooper T et al. (2000): Rapid detection of microorganisms in Blood Cultures of newborn infants utilizing an automated blood culture system. Pediatrics, 105 (3): 135-141.

25. Kupperman N, Walton EA (1999): Immature neutrophil in the blood smears of young febrile children. Arch.Pediatr Adolesc Med., 153 (3): 261-6.

26. Procianoy RS, Silviera RC (2004): The role of sample collection timing on interleukin-6 levels in early onset neonatal sepsis. J Pediatr (Rio J), 80 (5): 407-10

27. Marchant A, Alegre ML, Hakim A et al. (1995): Clinical and biological significance of interleukin-10 plasma levels in patients with septic shock. J Clin Immunol., 15 (5): 266-273. 